\title{
Conf-94106/--19
}

UCRL-JC-117141

\section{Development of a Portable Ambient Temperature Radiometric Assaying Instrument}

\author{
Anthony D. Lavietes \\ James H. McQuaid \\ Wayne D. Ruhter
}

This paper was prepared for submittal to the

1994 IEEE Nuclear Science Symposium and Medical Imaging Conference, Norfolk, Virginia,

October 30 - November 5, 1994

October 1994

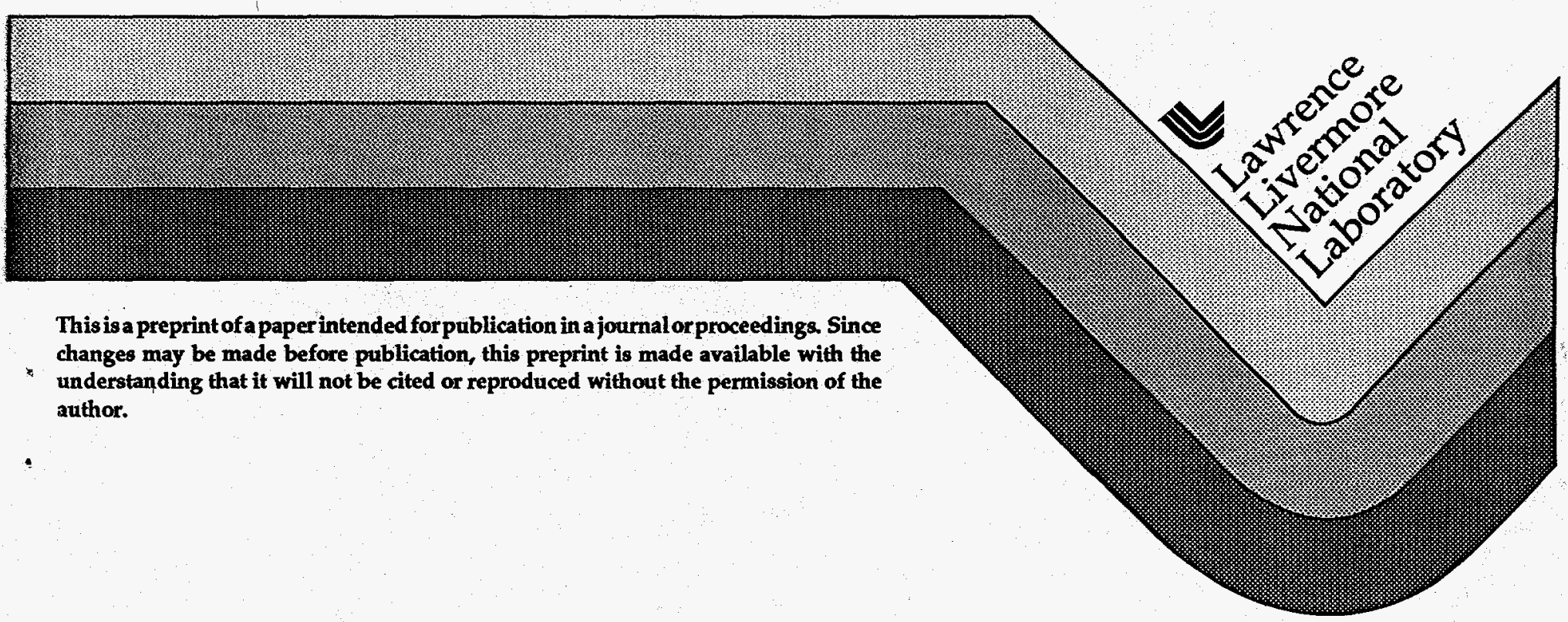


DISCLAIMER

This document was prepared as an account of work sponsored by an agency of the United States Government. Neither the United States Government nor the University of California nor any of their employees, makes any warranty, express or implied, or assumes any legal liability or responsibility for the accuracy, completeness, or usefulness of any information, apparatus, product, or process disclosed, or represents that its use would not infringe privately owned rights. Reference herein to any specific commercial products, process, or service by trade name, trademark, manufacturer, or otherwise, does not necessarily constitute or imply its endorsement, recommendation, or favoring by the United States Government or the University of California. The views and opinions of authors expressed herein do not necessarily state or reflect those of the United States Government or the University of California, and shall not be used for advertising or product endorsement purposes. 


\section{DISCLAIMER}

Portions of this document may be illegible in electronic image products. Images are produced from the best available original document. 


\title{
Development of a Portable Ambient Temperature Radiometric Assaying Instrument ${ }^{1}$
}

\author{
A.D. Lavietes, J. H. McQuaid, and W. D. Ruhter \\ Lawrence Livermore National Laboratory \\ T. J. Paulus \\ $\mathrm{EG} \& \mathrm{G}$ ORTEC
}

\begin{abstract}
There is a strong need for portable radiometric instrumentation that can accurately confirm the presence of nuclear materials and allow isotopic analysis of radionuclides in the field. To fulfill this need we are developing a hand-held, non-cryogenic, low-power gammaand X-ray measurement and analysis instrument that can both search and then accurately verify the presence of nuclear materials. We will report on the use of cadmium zinc telluride detectors, signal processing electronics, and the new field-portable instrument based on the MicroNOMAD Multichannel Analyzer from EG\&G ORTEC. We will also describe the isotopic analysis that allows uranium enrichment measurements to be made accurately in the field.

\section{INTRODUCTION}

This paper describes a truly field-portable instrument capable of search and verification of nuclear materials. The benefits of this work are realized in a wide spectrum of applications that include Arms Control, Nuclear Safeguards; Environmental Management, Emergency Response, and Treaty Verification. In addition, we characterize our instrument for use as an environmental screening tool by $\mathrm{X}$ ray fluorescence analysis of heavy metals.

Instrumentation for high-resolution spectrometry that is presently available for the applications described above is physically large, requires high power, and incorporates a liquid-nitrogen cooled detector assembly; these aspects limit its use in field applications and covert operations. Therefore, this work focuses on the use of an ambient temperature detector, low-power electronics, and hand-held operation.
\end{abstract}

\section{DETECTORS}

\section{A. Detector Selection}

There are currently no ambient temperature detectors known that provide both the high efficiency required to conduct a reasonable search, and the resolution necessary for

\footnotetext{
1 This work performed under the auspices of the U.S. Department of Energy by the Lawrence Livermore National Laboratory under Contract W-7805-Eng-48.
}

isotopic analysis. Developing an ambient temperature system to satisfy this need for analyzing radionuclides has been a challenge for a number of years. Most of this type of analysis work has been in conjunction with liquid nitrogen (LN) cooled germanium (Ge) detector systems in laboratories. Obviously, the logistics necessary to support a cooled $\mathrm{Ge}$ system has always been a problem outside the laboratory. Since our instrument is intended to provide both a search mode and an isotopic analysis mode of operation, a dual-detector system approach was adopted.

The first detector needed was one capable of high sensitivity searches. Sodium iodide (Nal) detectors have been used in this capacity for some time. These detectors are available in almost any size and configuration and will provide high enough resolution to determine the presence of radionuclides of possible interest. A system of this type is currently available from ORTEC (ScintiPack 296 and a NaI detector) and will be integrated with the MicroNOMAD to fulfill the needs of the search mode detector.

The detector to be selected for the confirmation, or verification mode of operation, had to meet several requirements. The most critical of the requirements. were moderate resolution and stable temperature characteristics. The resolution requirement was determined to be $<3 \%$ on ${ }^{57} \mathrm{Co}(122 \mathrm{keV})$. Three detector technologies met these requirements though only two were available in quantity; cadmium telluride (CdTe), and cadmium zinc telluride (CZT). The third technology, mercuric iodide, is not yet commercially available.

Due to the use of a modified High Pressure Bridgeman (HPB) crystal growth technique[1], very large CZT boules of excellent homogeneity can be grown. The addition of zinc to $\mathrm{CdTe}$ also has the benefits of both increasing the resistivity of the material several orders of magnitude and raising the bandgap from 1.47 to $1.6 \mathrm{eV}$. The higher resistivity allows for larger detectors and the requisite higher bias voltages necessary for improved charge collection. The higher bandgap reduces the bulk generated leakage current providing better ambient temperature operation. It was for these reasons that CZT was chosen for the isotopic analysis detector.

\section{B. Measurement Techniques}

It is important to discuss the method that is used to measure the Full Width at Half Maximum (FWHM) 
resolution. At this point in time, there exists several methods for determining FWHM, each resulting in different performance figures for equivalent devices. In an effort to remain consistent, the FWHM data presented in this paper will be calculated using absolute counts from the baseline. The peak amplitude of the energy line of interest will be divided by two, and the width of the pulse height distribution at that level will be used. This method of determining the FWHM takes into account the effects of low energy tailing from charge collection problem associated with the detector. Another paper[3], written by the authors and also presented at this conference, addresses this issue.

\section{CZT Detector Characteristics}

The first obstacle to overcome was the design of a low power, low noise, front end package that would optimize the performance of CZT detectors without the use of elaborate signal processing techniques. These techniques [e.g., Pulse Shape Discrimination (PSD), Charge Loss Correction, and Time-Variant Shaping] have all been applied successfully in various systems in the past, but they also bring with them additional power requirements, loss of counting efficiency, and other problems which prevent meeting our application criteria.

Many detectors of differing geometries were evaluated for charge transport problems -- such as slow components in the charge collection process.

The risetime data from a $2 \times 2 \times 2 \mathrm{~mm}$ CZT detector is shown in Fig. 1 and 2. A single charge collection waveform is shown in Fig. 1. This is a typical CZT charge pulse showing a fast risetime with characteristic slow charge collection at the top of the pulse. Fig. 2 is an average of 1000 charge collection risetime waveforms. These signals were taken from the output of a modified ORTEC 142PC charge sensitive preamp.

A pulse risetime distribution for this material shows a small percentage of slow charge collection components. The high resistivity of $\mathrm{CZT}\left(\cong 10^{11} \Omega \cdot \mathrm{cm}\right)$ allows for a higher bias

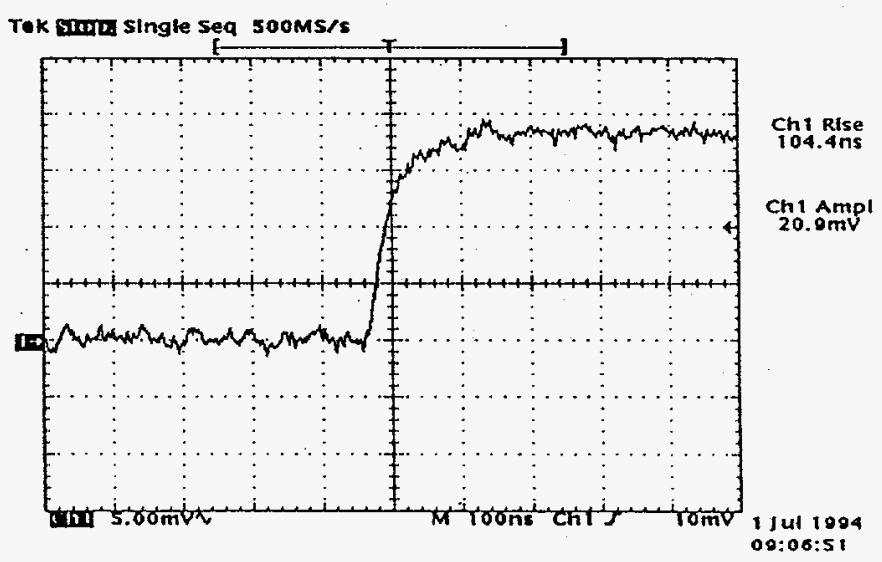

Fig. $12 \times 2 \times 2 \mathrm{~mm}$ CZT Detector Charge Pulse Risetime

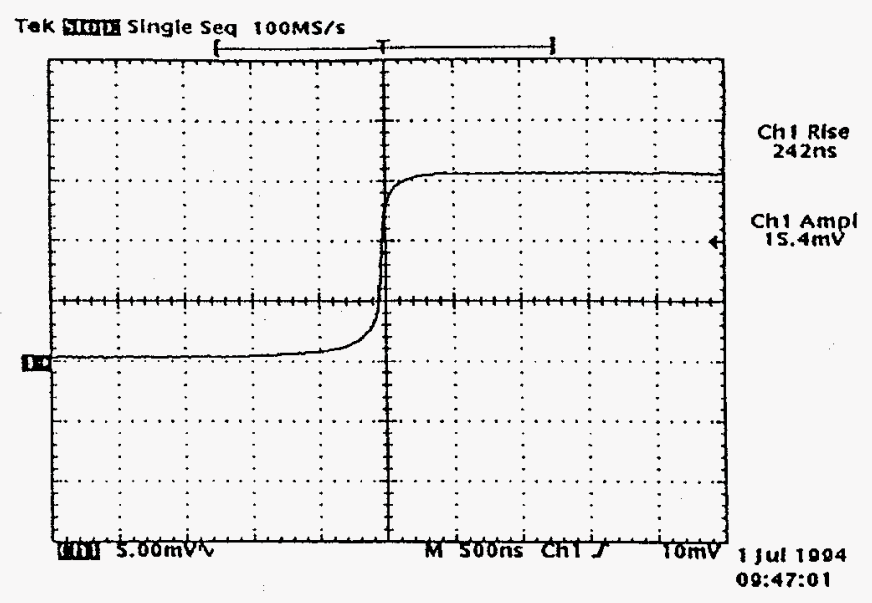

Fig. 2 CZT Charge Pulse Risetime

voltage and the subsequent higher electric field, which results in better charge collection.

This provides only a small overall percentage of slow charge collection components which, in turn, results in the poor response of CZT detectors to PSD techniques. The effect of PSD is to examine the risetime of each pulse and reject any that do not conform to a minimum risetime threshold. As can be seen in Fig. 1, this technique would be of negligible benefit. In practice, the application of PSD actually degrades the system performance. PSD typically resulted in the rejection of 60 to over $90 \%$ of the events, depending on the risetime threshold setting. Since the benefit of this technique with respect to resolution and low energy tailing for CZT is marginal, the largest observable result was a significant loss of efficiency.

Recent advances in CZT crystal-growth techniques have led to the fabrication of detectors of relatively large volumes exhibiting the required energy resolution with high yields that result in low cost. This has made it practical to pursue development of this field instrument. It is now reasonable to expect a resolution of $<3 \%$ on the $122 \mathrm{keV}$ peak of ${ }^{57} \mathrm{Co}$ with a $5 \times 5 \times 5 \mathrm{~mm}$ CZT detector. Significantly, this good resolution was achieved on a relatively large volume detector without pulse shape discrimination or other schemes that drastically reduce the photopeak efficiency, as previously mentioned.

\section{ELECTRONICS}

\section{A. CZT Electronics}

Even with improved CZT material, noise (primarily shot noise), poor hole-mobility, and charge trapping contribute to less than ideal energy resolution. Therefore, the preamplifier and filter amplifier have been designed to minimize these effects. All front-end electronics currently use integrated circuit operational amplifiers (IC opamps) and will use surface-mount technology in the final version.

The prototype system that has been designed and tested is shown in Fig. 3. This first prototype design has limited 


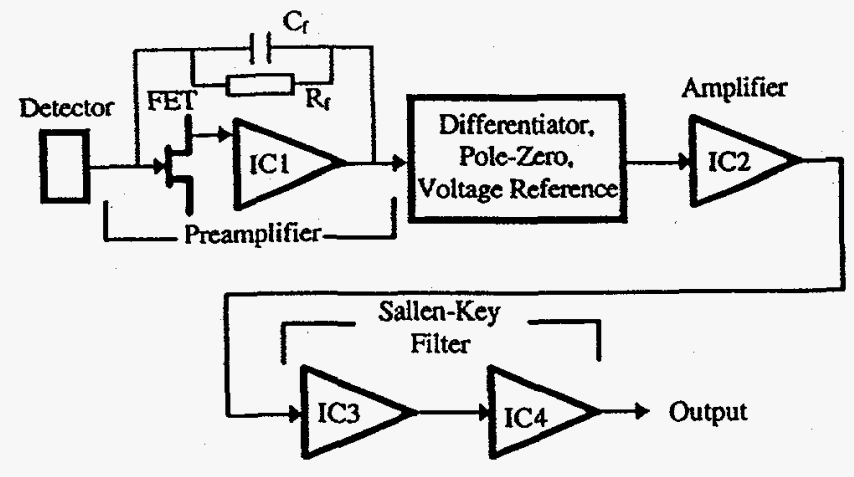

Fig. 3. Block Diagram of the Prototype CZT System

correction for poor charge collection, but achieves excellent reduction of spectral noise.

An example of a spectrum obtained using a ${ }^{57} \mathrm{Co}$ source and a $5 \times 5 \times 5 \mathrm{~mm}$ CZT detector is shown in Fig. 4. The energy resolution of the $122 \mathrm{keV}$ peak is $3.32 \mathrm{keV}$ FWHM. The electronic contribution (pulser resolution without the detector) is $1.2 \mathrm{keV}$ FWHM using $0.5 \mu \mathrm{s}$ shaping ( $1.0 \mu \mathrm{s}$ peaking). This monolithic design was able to achieve good resolution (10\% better than commercial instrumentation) and low power consumption $(600 \mathrm{~mW})$. This low power consumption is compatible with the AA batteries used in the MicroNOMAD.

A low noise field-effect transistor (FET) was selected for optimization of series and parallel noise, front end gain, and rise time. Good stability is achieved by running a $400 \mathrm{MHz}$ opamp (IC1) in an open loop configuration with DC feedback for stability. This IC is a critical component that achieves the necessary combination of high bandwidth and low noise. In previous designs, this portion of the preamplifier was usually a hybrid circuit, which is physically larger and has higher power consumption. Power is further reduced in this design by running $\mathrm{ICl}$ on a single ended supply $(+12 \mathrm{~V})$. The preamplifier sensitivity is $20 \mathrm{~V} / \mathrm{pC}$ and has a rise time of $24 \mathrm{~ns}$.

The amplifier stage is voltage sensitive and is composed of a low noise, high bandwidth opamp preceded by a differentiator and pole-zero compensation network. Coarse and fine gain controls allow adjustment for processing ${ }^{241} \mathrm{Am}$ $(60 \mathrm{keV}),{ }^{57} \mathrm{Co}(122 \mathrm{keV})$, or ${ }^{137} \mathrm{Cs}(662 \mathrm{keV})$ full scale. Further power reduction is accomplished by establishing a voltage reference that allows the remaining circuitry to run on the single-ended supply.

A two-stage Sallen-Key filter was designed using opamps IC3 and IC4. This circuit has two pairs of complex poles and was found to be very stable. This circuit also has a sharp noise-corner response, which is very important in reducing parallel noise and at the same time minimizing ballistic deficit due to the slow charge collection of the detector. The shaping time of this filter is $0.5 \mu \mathrm{s}$ ( $1.0 \mu \mathrm{s}$ peaking time). The optimum shaping time for the electronics is 4-6 $\mu \mathrm{s}$, based on the capacitance of the detector and other circuit

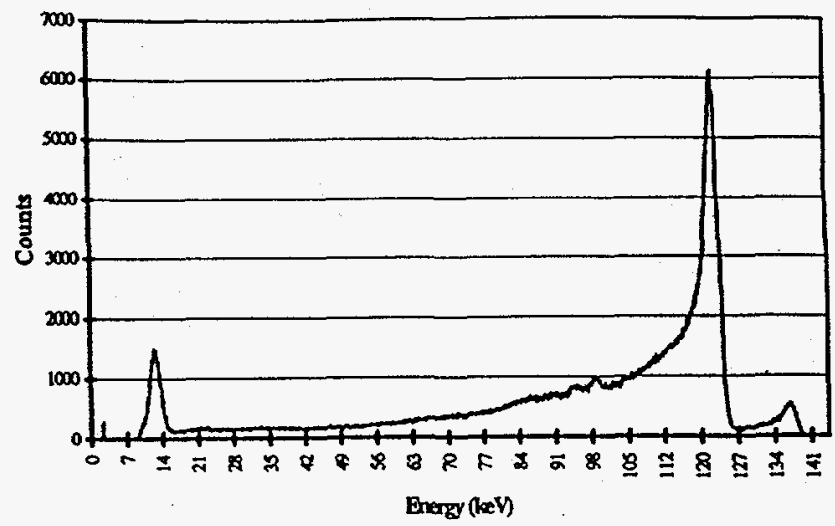

Fig. 4 Energy Spectrum of ${ }^{57} \mathrm{Co}$ Using a $5 \times 5 \times 5 \mathrm{~mm}$ CZT Detector

parameters. However, detector charge trapping phenomena has a pronounced effect on resolution, and as a result, the filter time constant must be reduced to $0.5 \mu \mathrm{s}$. This reduces the amount of low-energy tailing and is based on the characteristics of many CZT detector measurements. These measurements have shown that trapping can add $2-3 \mathrm{keV}$ (in quadrature) to the resolution if the filter time constant is increased to $1 \mu \mathrm{s}$.

The second stage of the filter was designed with a higher $Q$ than the first stage This design achieves improved performance and results in a nearly Gaussian output pulse shape.

\section{B. Portable Multichannel Analyzer}

Both the high-efficiency search detector and the highresolution analysis detector require a multichannel analyzer. The MicroNOMAD is a $1.5-\mathrm{lb}$., battery powered, portable gamma-ray spectrometer developed especially for this application. The block diagram is shown in Fig. 5. The unit consists of an amplifier suitable for $\mathrm{NaI}, 2 \mathrm{kADC}$, spectrum stabilizer, microprocessor and memories, as well as parallel, RS-232, and RS-485 ports. The internal amplifier can be bypassed when using CZT with its optimized amplifier described above. Both the ScintiPack NaI probe and the CZT probe connect to the MicroNOMAD using a single cable. The unit is small, only $7 \times 7 \times 21 \mathrm{~cm}$, and requires $<1.5$ W. Its $8 \mathrm{AA}$ batteries will operate the unit for over 8 hours.

Field-mode operation allows the MicroNOMAD to record multiple spectra at the push of a button or the scan of a bar code. Each acquisition is performed in accordance with the preset values of real time, live time, gross ROI counts, or peak ROI counts. Up to 63512 -channel spectra can be saved. At the end of each acquisition, the spectral data, start time, real time, live time, and up to 16 characters read by the bar code can be stored.

Any computer can be connected to the MicroNOMAD using the built-in parallel port. In-field automatic nuclide identification is easy using analytical software specially 


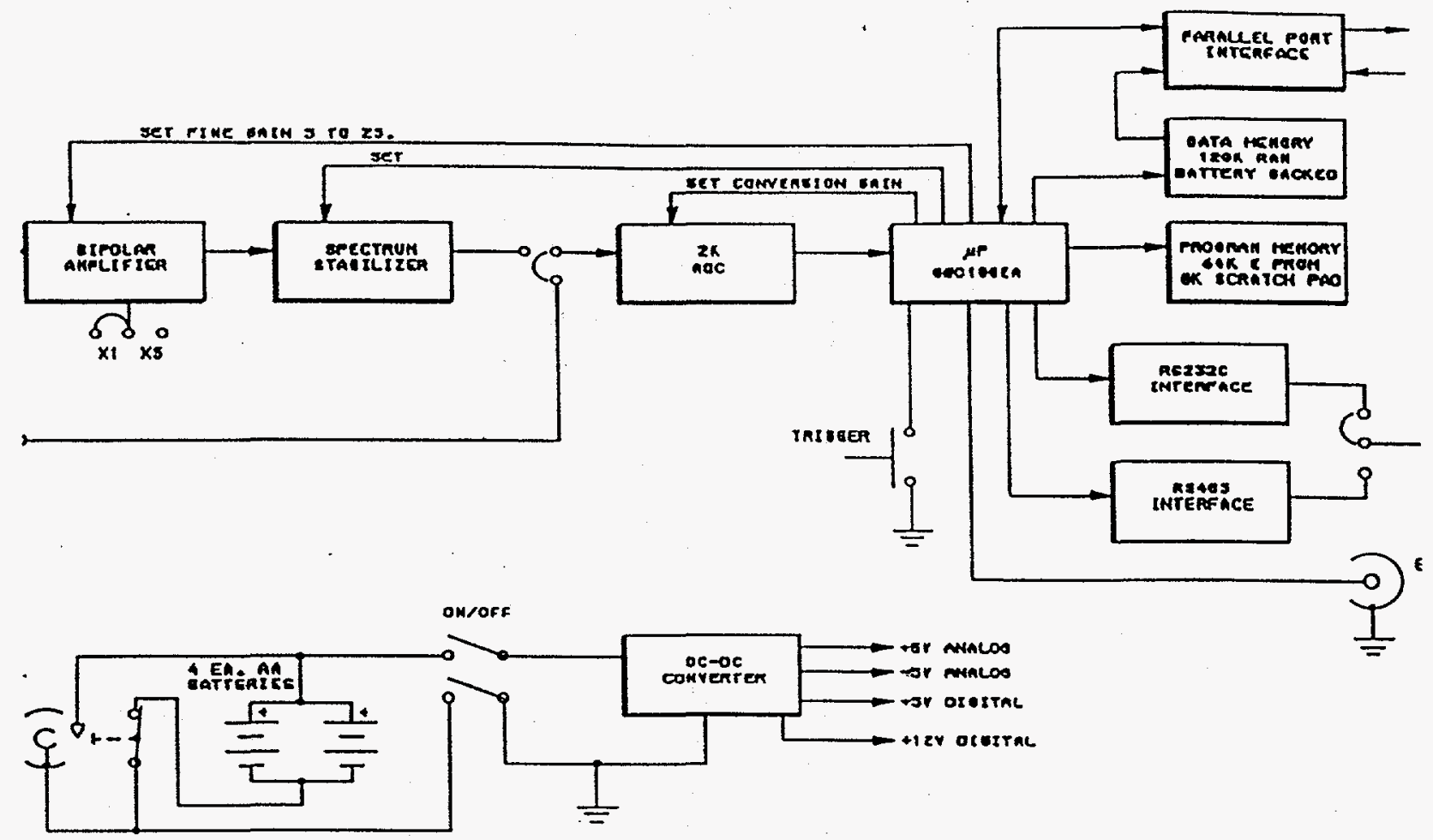

Fig. 5 MicroNOMAD Block Diagram

designed for NaI detectors. Multipoint energy and efficiency calibration is also provided.

\section{APPLICATIONS}

\section{A. Uranium Analysis}

Isotopic analysis of uranium has been performed using $2 \times 2 \times 2 \mathrm{~mm}$ and $5 \times 5 \times 5 \mathrm{~mm}$ CdZnTe detectors with samples of

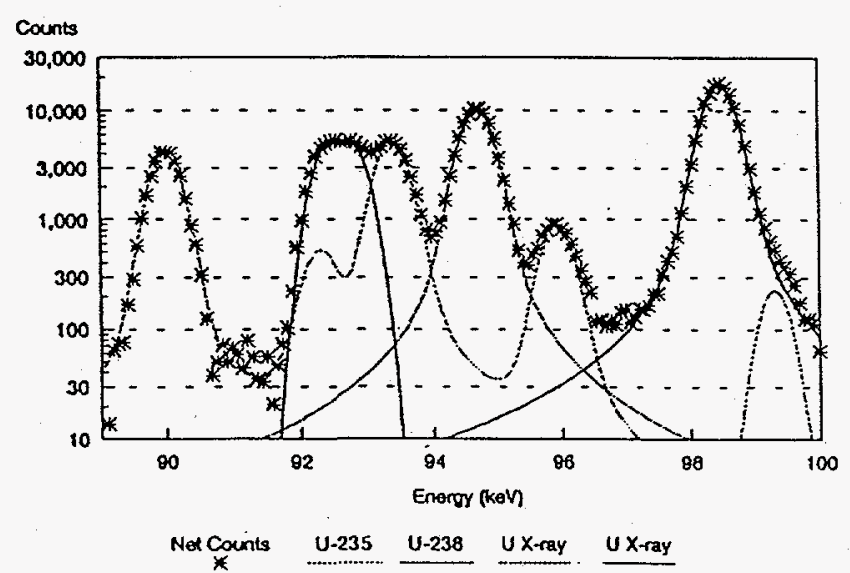

Fig. 6 Uranium Spectrum with High Resolution Detector $(484 \mathrm{eV}$ at $100 \mathrm{keV})$
${ }^{235} \mathrm{U}$ enrichments ranging from $3 \%$ to $50 \%$. We use a spectrum analysis technique incorporated in GRPANL[2] that fits ${ }^{235} \mathrm{U},{ }^{238} \mathrm{U}$, and $\mathrm{U} \mathrm{K} X$-Ray response profiles to data in the 89- to $100-\mathrm{keV}$ region of a gamma-ray spectrum. From the relative magnitudes of the ${ }^{235} U$ and ${ }^{238} U$ profiles, we determine the ${ }^{235} \mathrm{U}$ enrichment with an accuracy of about $10 \%$ with CdZnTe detectors.

The result of using GRPANL is shown in Fig. 6 and 7 . The analyzed output using spectra from a high resolution

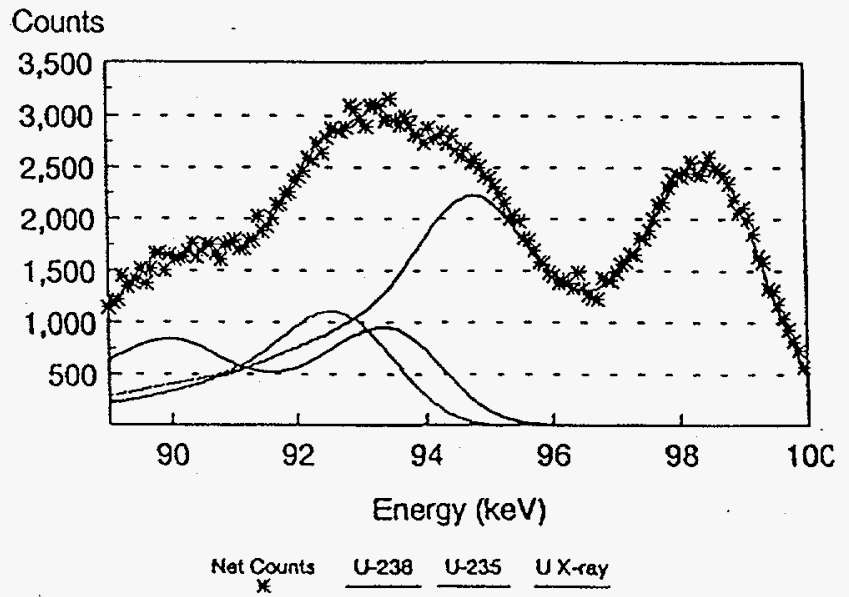

Fig. 7 Uranium Spectrum with CZT Detector $(2.9 \mathrm{keV}$ at $100 \mathrm{keV}$ ) 
Table 1

GRPANL Measurement Data

\begin{tabular}{|c|c|c|}
\hline $\begin{array}{c}\text { Declared } \\
{ }^{235} \mathrm{U}^{238} \mathrm{U}\end{array}$ & $\begin{array}{c}\text { Measured } \\
\pm \% \mathrm{RSD}\end{array}$ & $\begin{array}{c}\text { Measurement } \\
\text { Time (hours) }\end{array}$ \\
\hline 0.0314 & $0.0302 \pm 16.8$ & 12 \\
\hline 0.0528 & $0.0557 \pm 12.5$ & 6 \\
\hline 0.1136 & $0.1116 \pm 4.6$ & 6 \\
\hline 0.9997 & $1.0093 \pm 13.7$ & 15 \\
\hline 3.166 & $4.42 \pm 36.3$ & 15 \\
\hline
\end{tabular}

*Relative Standard Deviations (RSD) were determined from the fits to the net counts in the spectra.

Low Energy Photon Spectrometer (LEPS) detector is shown in Fig. 6. The enrichment is determined by the ratio of the ${ }^{235} \mathrm{U}$ and ${ }^{238} \mathrm{U}$ peaks located between 92 and $94 \mathrm{keV}$. In this case, the sample was $10 \%$ enriched uranium. The same sample was analyzed by the $5 \times 5 \times 5 \mathrm{~mm}$ CZT. detector system and the resulting GRPANL data is shown in Fig. 7. The resolution of the CZT system is markedly worse (2.9 keV for CZT and $484 \mathrm{eV}$ for the LEPS detector), but the software was still able to fit the peaks accurately enough to give the same result. The CZT system was then used to analyze several uranium samples of differing enrichments. This data is shown in Table 1. The amount of time necessary for spectral acquisition was extremely long due to the fact that 1 gram samples were used. A commercial instrument using this technology would certainly benefit from larger detectors resulting in better efficiency. The enrichment analysis required a detection system resolution of $<3 \%$ at $100 \mathrm{keV}$ to be able to adequately discriminate between peaks that were closely spaced in terms of energy.

\section{B. X-ray Fluorescence}

The CZT detection system was used to characterize lead by X-ray fluorescence. A lead sample was illuminated by a ${ }^{57} \mathrm{Co}$ source. Fig. 8 shows the resulting spectrum and verifies the ability of this system to be used as an

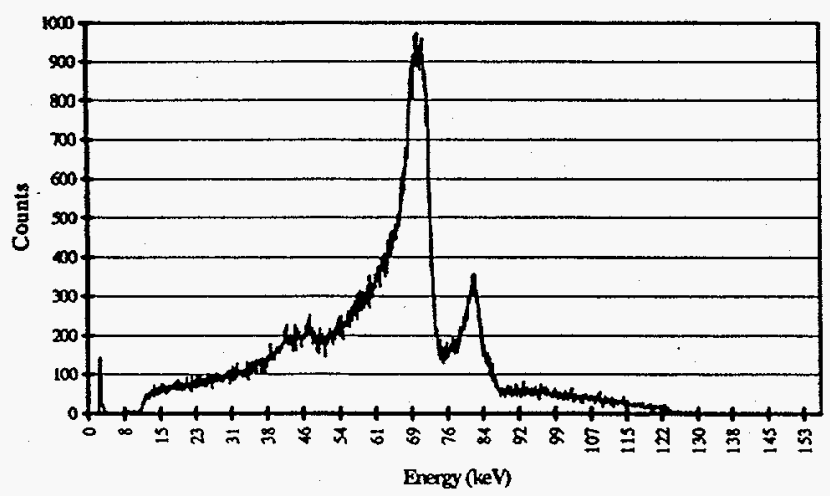

Fig. 8 Lead X-ray Fluorescence using $122 \mathrm{keV}{ }^{57} \mathrm{Co}$ and a $5 \times 5 \times 5 \mathrm{~mm}$ CZT Detector environmental sample screening tool. Energy resolution is $2.8 \mathrm{keV}$, FWHM -- typically that obtained for ${ }^{241} \mathrm{Am}(60$ $\mathrm{keV}$ ). A desirable feature of this technology, with respect to heavy metal X-rays, is that $100 \%$ absorption takes place in relatively small volume detectors.

\section{Conclusions}

We have successfully shown that ambient temperature radiometric assaying can be performed with current technology. A truly portable instrument has been developed to meet the needs of several important applications. Immediate applications include Safeguards and Environmental Management, illustrated by the successful demonstration of this instrument for actinide and heavy metal characterization.

During the course of this work, it was determined that a standard method was needed for characterization of $\mathrm{CdTe}$ and CZT detectors. We have addressed this by developing a proposed standard based on the IEEE Standard Test Procedures for Semiconductor X-ray Energy Spectrometers (ANSU/IEEE Std. 759-1984).

While performing detector characterizations, it was noted that charge trapping appears to be the dominant factor in resolution (FWHM) degradation. This is even true in detectors that have significant shot noise as a result of high leakage currents ( $\geq 40 \mathrm{nA})$. This data supports the need for more research to better understand charge transport mechanisms in this material. In addition, signal processing techniques will be applied in order to address the charge collection problems associated with these ambient temperature detectors.

\section{REFERENCES}

[1] E. Raiskin and J. F. Butler, "CdTe Low Level Gamma Detectors Based on a New Crystal Growth Method," IEEE Transactions on Nuclear Science, vol. 35 no. 1, pp. 81-84, February 1988.

[2] R. Gunnink, W.D. Ruhter. and J.B. Niday, "GRPANL: A Suite of Computer Programs for Analyzing Complex Ge and Alpha-Particle Spectra," Lawrence Livermore National Laboratory, UCRL-53861, Vol. 1, May 1988.

[3] A. D. Lavietes and J. H. McQuaid, "Characterization Inconsistencies in CdTe and CdZnTe Gamma-Ray Detectors," IEEE Nuclear Science Symposium and Medical Imaging Conference, Norfolk, Virginia, November 1994. 